\title{
Actividades de innovación en la materia de Diseño de Experimentos para máster universitario
}

\author{
Vicente Chirivella-González, Rosa María Alcover-Arándiga, Bernardo J. Richart-Solá \\ Departamento de Estadística e Investigación Operativa Aplicadas y Calidad. Universitat Politècnica \\ de València (España). Email \{vchirive, ralcover, brichart\}@upv.es
}

\begin{abstract}
Resumen
En este trabajo analizamos el efecto que la incorporación de las metodologías activas ha tenido sobre el aprendizaje en la materia de Diseño de Experimentos (DOE) impartida en el master de Análisis de datos, Mejora de procesos y Toma de decisiones en la Universitat Politècnica de València (UPV). Las nuevas actividades introducidas en la materia de DOE se basan en la realización de experimentos en el aula, experimentos sencillos, que permiten desarrollar habilidades en el alumnado y trabajar competencias necesarias para la práctica real del DOE en la industria. Además de la planificación, ejecución y análisis de los experimentos, nuestros estudiantes trabajan las competencias de comunicación efectiva, tanto oral como escrita $y$ de trabajo en equipo.
\end{abstract}

Palabras clave: Diseño de Experimentos; Práctica de laboratorio; Innovación educativa; Metodologías activas; Dinamización del aula; Competencias.

\section{Introducción}

La sociedad actual demanda cada día con mayor interés profesionales en el análisis y tratamiento de datos con el fin de conseguir una mayor información que guíe sus procesos de toma de decisiones, prueba de ello son las numerosas titulaciones ofrecidas por la universidad en materia de ciencia de datos y big data. Por otra parte, en el área de la ingeniería, las técnicas estadísticas de Diseño de Experimentos (DOE) se han perfilado como una de las herramientas más relevantes en el incremento de la calidad y productividad tanto de los productos como de los procesos productivos Romero y Zúnica (2004), Montgomery (2012).

Durante el curso 2009/2010 y previendo esta situación, la UPV puso en marcha el máster universitario en Ingeniería de Análisis de datos, Mejora de procesos y Toma de decisiones, 
incluyendo una materia de DOE organizada en dos asignaturas. La materia de DOE, ubicada en el módulo de Mejora de Procesos del máster, se compone de las asignaturas DOE I (DOE básico) y DOE Avanzado, de 3 créditos ECTS cada una de ellas. En DOE I, se parte de conocimientos básicos y elementales, llegando a abordarse en DOE Avanzado aspectos de elevado nivel de complejidad. De esta forma, la visión de DOE del futuro profesional en análisis de datos, mejora de procesos y toma de decisiones, es muy completo.

Desde la puesta en marcha del master hasta la actualidad (curso 2017/2018) los contenidos teóricos de la materia no han variado sustancialmente. El cambio más importante ha tenido lugar en los objetivos perseguidos, en la metodología docente utilizada y en el sistema de evaluación adoptado (Fernández, 2006). Estos cambios repercuten en mejores resultados del alumnado, en un mayor rendimiento de la materia y en una mayor satisfacción tanto del alumnado como del profesorado implicado (González, 2013). A lo largo de las seis ediciones del máster, la materia de DOE se ha ido configurando y adaptando con el objetivo de conseguir un mejor cumplimiento de las competencias requeridas tanto por el propio máster como por la UPV. Para ello, en primer lugar se ha reducido el tiempo dedicado a la exposición teórica del profesor, estrategia necesaria pero que difícilmente por sí sola fomenta el aprendizaje autónomo del alumnado, GIMA (2008). Además, siguiendo a diferentes autores (Peiró-Signes y Segarra-Oña, 2015), se han introducido nuevas actividades que intentan dinamizar la actividad en el aula y garantizar el desarrollo de las competencias específicas y transversales (Proyecto institucional de las competencias transversales UPV). En nuestra opinión, el cambio más profundo en la materia durante este periodo, tanto en la organización, metodología y evaluación, está ligado precisamente a la adquisición de las competencias por parte de los estudiantes.

Hoy en día, el profesorado universitario se enfrenta al reto continuo de proponer nuevas actividades en el aula con el fin de motivar y promover la participación activa de su alumnado. Además, estas actividades suelen variar su nivel de complejidad según el perfil del alumno al que van dirigidas. En la experiencia que presentamos en este trabajo, al tratarse de una materia de máster, ha requerido una considerable carga de trabajo por parte de los dos profesores de la materia y del técnico de laboratorio implicado (Villa y Poblete, 2007), debido a la heterogeneidad en la procedencia, perfil y nivel del alumnado.

Los profesores hemos constatado que, tras la modificación de la metodología docente y la incorporación de las nuevas actividades en las asignaturas de DOE, los resultados obtenidos por los alumnos matriculados en la materia desde el curso 2009/2010, son significativamente mejores, además de trabajar en la actualidad nuevas competencias que se habían pasado totalmente por alto hace unos años. En nuestra opinión, siguiendo la nueva metodología, se incrementa y mejora del aprendizaje, fomentando el que los alumnos en un futuro cercano lleguen a ser mejores profesionales en su campo. 
Por todo ello, en este trabajo proponemos una metodología activa para impartir las dos asignaturas de la materia de DOE incluidas en el máster universitario de Ingeniería de Análisis de datos, Mejora de procesos y Toma de decisiones, impartido por la UPV. Esta metodología se recoge en Chirivella, Alcover y Richart (2018), y se basa en la introducción de diferentes actividades de trabajo en equipo, con material especialmente diseñado para ello, que permiten dinamizar el aula durante sesiones de seis horas de clase con los alumnos, motivando la participación activa de los estudiantes, garantizando la adquisición de competencias y, en definitiva, facilitando y fomentando el aprendizaje. Nuestro objetivo es conseguir un aprendizaje activo, significativo. En el presente trabajo aplicamos nuestra propuesta al diseño de experimentos orientados a la construcción de un autogiro, con el fin de optimizar su tiempo de vuelo.

\section{Metodología propuesta}

Con los objetivos planteados en el apartado anterior y desde las asignaturas implicadas, los profesores implicados hemos propuesto actividades centradas en el diseño y realización de experimentos que se puedan implementar fácilmente en el aula informática, tanto en tiempo como en los recursos necesarios. A su vez, y al tratarse de una materia impartida en máster, estos experimentos deben ser lo suficientemente elaborados para cubrir el desarrollo y posterior evaluación de un gran número de competencias transversales en los alumnos. En nuestra opinión, este tipo de actividades constituyen el nexo entre la teoría, la práctica y el entorno real de trabajo de un futuro profesional.

De los posibles experimentos planteados por el profesorado, hemos escogido el experimento de la construcción de un autogiro por ser una experiencia muy visual, didáctica y que permite barajar múltiples soluciones alternativas. Además este es un experimento sumamente sencillo y económico, pues no requiere una gran inversión en material de trabajo ni en espacio en el que llevar a cabo la experimentación. Esta actividad de experimentación facilita a los estudiantes, además del desarrollo de las competencias generales y específicas de la asignatura, trabajar la competencia transversal de innovación, creatividad y emprendimiento, proporcionando diseños y ensamblados novedosos de un proceso.

\section{El experimento del autogiro}

El objetivo de esta actividad es utilizar el diseño estadístico de experimentos para diseñar un autogiro de papel que tarde el mayor tiempo posible en llegar al suelo, tras lanzarlo desde una determinada altura. En la escala adecuada, se trata de una forma muy sencilla y 
barata de fabricar drones (engineering.com, 2013) encargados de filmación de imágenes, cartografía, emergencias, logística, seguridad,...

Para ello es necesario conocer la relación que existe entre una variable respuesta, el tiempo de vuelo del autogiro, y los factores de diseño considerados para la misma, con el fin de poder fijar sus valores y alcanzar un cierto objetivo, maximizar el tiempo de vuelo. Responder a todo ello implica tener que lidiar con los problemas "logísticos" de realizar el experimento, por ejemplo, la forma de medir las variables, escoger los niveles de los factores, organizar las experiencias de acuerdo a un presupuesto limitado, llevarlas a cabo sin exceder dicho presupuesto y medir la variable respuesta con el instrumento adecuado.

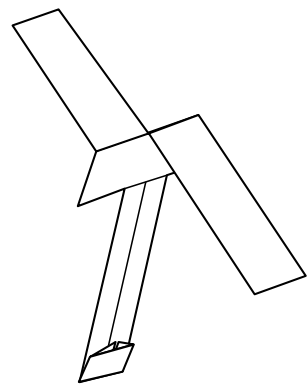

Fig. 1 Autogiro de papel, listo para volar.

Por comenzar a concretar el problema general planteado, resaltar que en la propia formulación del objetivo viene especificada la variable respuesta o característica de calidad a optimizar, pues podrían considerarse como tales además del tiempo de vuelo, la distancia entre el punto de aterrizaje y la vertical del punto de lanzamiento, el tiempo que dura el periodo transitorio (hasta que se estabiliza el movimiento),... entre otras. La característica elegida es el tiempo que dura el vuelo del autogiro, y deseamos que dicho tiempo sea lo más grande posible.

Por otra parte, existen muchos factores de diseño que determinarán el tiempo de vuelo de nuestro autogiro. Algunos tienen que ver con magnitudes físicas del propio autogiro (Figura 2). Los factores propuestos para analizar su posible efecto sobre el tiempo de vuelo serían: el tipo de papel (gramaje de 80 y $120 \mathrm{gr} / \mathrm{m}^{2}$ ), la longitud y la anchura del cuerpo, así como la longitud y la anchura de las alas (cuyos valores máximos vienen dados por la plantilla que proporcionamos al alumno), la carga transportada por el autogiro (simulado con uno o más clips disponibles), el pegado del cuerpo con celo para disminuir la resistencia al aire (con o sin celo), la posible rigidez de las alas (conseguida mediante palillos), y finalmente la presencia de publicidad (con o sin publicidad).

El modelo de autogiro de papel que se muestra a continuación (Figura 2) es la base para realizar los experimentos. Este diseño presenta unas líneas de diferente color o trama que 
pueden ser utilizadas para definir los niveles de algunos factores. Las líneas de color rojo indican que debemos cortar el papel, y las de trazos que debemos doblar. Las líneas verticales azules de los extremos son opcionales para conseguir una mayor o menor longitud del ala (izquierda) o una mayor o menor longitud del cuerpo (derecha).

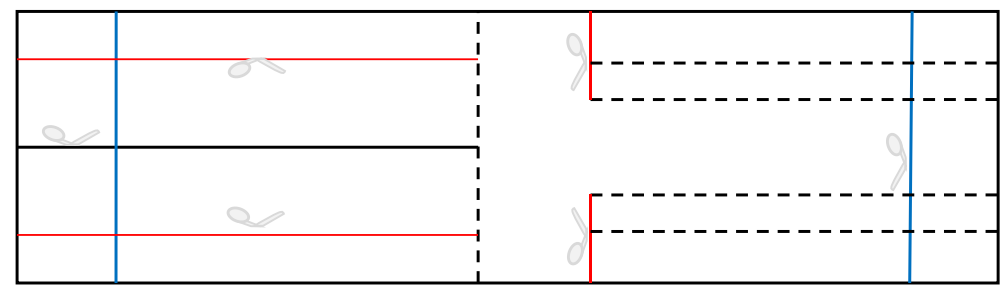

Fig. 2 Plantilla del autogiro de papel

Los alumnos, trabajando en equipo, seleccionarán los posibles factores de diseño del autogiro más relevantes, de entre los propuestos, añadiendo aquellos que su experiencia previa con aviones de papel quieran añadir, como timones, alerones,... Todos estos factores proporcionan multitud de posibilidades de diseño en la construcción del autogiro.

El trabajo a realizar por el alumno se divide en cinco partes. La primera parte corresponde a la toma de contacto con el material para construir el autogiro de papel y un primer enfrentamiento a las cuestiones logísticas de su implementación. En la segunda y tercera se realiza el análisis del problema, obteniendo en primer lugar un cierto conocimiento básico sobre el mismo, que llevará en la siguiente parte al planteamiento y ejecución del trabajo con el análisis completo del problema. En la cuarta parte los alumnos escribirán una memoria que documentaría toda la actividad realizada, y en la quinta prepararán dos presentaciones, una dirigida a sus compañeros de clase, y otra que se expondría a sus jefes en la "empresa". Con cierto detalle:

1. Lo primero que deben hacer los alumnos es recoger el material para realizar el experimento: tijeras, regla, clips y cronómetro. Con una plantilla del autogiro que les proporcionamos realizan de forma individual un primer diseño, en la que su intuición como antiguos constructores de aviones de papel les lleva a construirlo y a probar sus capacidades de vuelo, que quedan anotadas en el propio cuerpo del autogiro. Una vez hecho esto se les invita a formar grupos de trabajo, en el que cada componente aporta el material de trabajo y las conclusiones preliminares conseguidas del vuelo de su primer autogiro. Como se les pide encontrar seis factores que posiblemente afecten al tiempo de vuelo del autogiro, así como el rango de los niveles o las variantes que le corresponderían a cada factor, emplean algo de tiempo en comparar los diseños individuales y extraer alguna conclusión. Los miembros del grupo se organizan informalmente para afrontar los problemas que aparecen.

2. Transcurrido un tiempo conveniente, los equipos deciden los factores a analizar, entre los que puede que se encuentra la publicidad, como forma de asegurar la presencia de un factor 
que se conoce a priori que no influirá en el tiempo de vuelo. En este momento deberían plantear lo que se conoce como un diseño de cribado, que permite determinar con un número mínimo de pruebas cuáles de los seis factores propuestos son los más relevantes para explicar tanto el promedio como la varianza del tiempo de vuelo, y las interacciones que existen entre ellos. Los grupos indican al profesor el número de plantillas necesarias para realizar las pruebas y las facilitamos, con la advertencia de que se trata de un recurso limitado, estableciendo la comparación con la práctica real en una empresa. Con el comienzo de la realización de las pruebas suelen aparecer cambios en la forma de proceder en ellas. Lo mismo ocurre con los roles y las tareas de cada miembro del equipo que ellos mismos se han asignado. Finalmente se realiza el análisis de los datos y se obtienen las conclusiones.

3. Con los resultados preliminares los alumnos deben proponer un nuevo diseño del experimento que permita ensayar los factores más relevantes encontrados, a los niveles y variantes que resulten adecuados, con el menor número de pruebas posible y con objeto de realizar el estudio definitivo del problema del autogiro. Los alumnos planifican y realizan los correspondientes experimentos, y analizan la naturaleza de la relación de los factores seleccionados con el promedio del tiempo de vuelo. Con estos mismos datos repiten los análisis, pero ahora con la varianza del tiempo de giro. Una vez explicado el proceso, los alumnos pueden diseñar el autogiro a voluntad, por lo que determinan los valores de los factores para maximizar el tiempo de vuelo tras ser lanzado desde 4 metros de altura. Para comprobar que todo lo obtenido y previsto es correcto, cada equipo realiza un experimento confirmatorio final en las condiciones operativas propuestas por ellos como óptimas.

4. Con el análisis estadístico de los datos ya realizado, los estudiantes deben documentar en una memoria final escrita todo el proceso de toma de decisión, los experimentos planteados, los resultados obtenidos y las conclusiones que se derivan del trabajo realizado. El enfoque de este documento o memoria es el formal de cualquier trabajo académico o de investigación.

5. Como conclusión del trabajo los equipos preparan dos exposiciones orales. La primera, con una duración de 18 minutos, tiene como objetivo explicar el documento final a sus compañeros, detallando los diseños seleccionados y el motivo de las soluciones adoptadas a los problemas encontrados durante la experimentación. En la segunda presentación, que tiene una duración de 8 minutos máximo, los estudiantes deberían explicar a los supuestos responsables de la empresa aeronáutica (a la que ofrecieron sus servicios y productos) las conclusiones y las acciones propuestas sobre el proceso de fabricación de los autogiros. La presentación que finalmente realizará cada equipo será escogida al azar entre las dos realizadas, y terminará con un turno de preguntas, tanto de compañeros como de profesores, con el fin de aclarar las cuestiones necesarias. 
Con la realización de estas actividades, los estudiantes ejercitan y desarrollan las competencias que deben adquirir en el curso (http://www.upv.es/contenidos/COMPTRAN/). Como es natural, del listado de competencias, la primera a desarrollar sería la CT13 Instrumental específica, en este caso, el uso de las herramientas estadísticas e informáticas necesarias para el ejercicio profesional. Así, para resolver el problema planteado del tiempo de vuelo del autogiro, los alumnos han seleccionado las herramientas estadísticas más adecuadas tales como el diseño de experimentos, el análisis de la varianza, los modelos de regresión y técnicas de optimización, integrándolas adecuadamente con el fin de conseguir el objetivo propuesto, maximizar el tiempo de vuelo. Además han utilizado las herramientas informáticas que implementan las técnicas estadísticas mencionadas, por ejemplo Statgraphics centurion, $S P S S, R$, y los programas ofimáticos para la elaboración del documento final y de las presentaciones (en general Excel, Word y PowerPoint). Por todo ello, con el trabajo del autogiro se ha llegado al nivel 3 de dicha competencia transversal. Esta competencia se evalúa mediante la nota obtenida en la memoria del trabajo, reconvirtiéndola en uno de los cuatro niveles posibles (No alcanzado, En desarrollo, Adecuado, Excelente).

La competencia transversal CT01 Comprensión e integración, también debe ser evaluada en la materia, por ser punto de control en el máster. Esta competencia se trabaja y evidencia en diversas fases del trabajo de los estudiantes en la actividad propuesta. Así, el diseño del autogiro escogido por los alumnos es una evidencia de la comprensión del objetivo y del funcionamiento del mismo; los diseños de experimentos utilizados son evidencia de haber entendido en cada caso la necesidad de establecer relación entre el tiempo de vuelo y los factores propuestos, de acuerdo al valor establecido de sus niveles y/o variantes. Además los alumnos integran contenidos y conceptos impartidos en diferentes asignaturas, facilitando la coordinación entre materias. Las evidencias de la comprensión e integración de estos contenidos y conceptos aparecen tanto en la redacción de la memoria del trabajo como en sus posteriores exposiciones. En estas, se establecen relaciones de causa-efecto, se expresan sus ideas y se generan las conclusiones, tal como indica el nivel 3 de dicha competencia. Esta competencia se evalúa mediante la rúbrica que tiene establecida para ello la UPV.

Además de la competencia transversal propia de la asignatura y de la competencia de compresión e integración, en esta actividad se han trabajado y desarrollado otras competencias, con la posibilidad de evaluarlas, si la ERT lo desea. Estas competencia son: CT02 Aplicación y pensamiento práctico, CT03 Análisis y resolución de problemas, CT08 Comunicación efectiva (tanto en su forma oral como escrita), CT09 Pensamiento crítico, CT06 Trabajo en equipo y liderazgo y CT12 Planificación y gestión del tiempo. 


\section{Resultados}

En nuestra opinión, los resultados obtenidos con este tipo de actividades son muy satisfactorios, siendo muy positivo el cambio de trabajo teórico a la actividad de experimentación al igual que el cambio en la forma de evaluación del alumno. Este hecho puede cuantificarse de forma objetiva por el mayor porcentaje de aprobados y la nota media del curso, que ha aumentado, como se muestra más adelante. Adicionalmente, existen otras cuestiones de carácter más subjetivo que animan al profesorado implicado a declarar el éxito del cambio, como son las opiniones de satisfacción manifestadas por los alumnos al terminar la sesión de trabajo en el laboratorio, tras la realización de la actividad. La observación de la actividad de experimentación durante su desarrollo ha mostrado una actitud totalmente participativa y muy positiva para los estudiantes. La resolución del problema, cuestión que los alumnos confirman en la realización del experimento final, les proporciona una sensación de cierre de la sesión con la evidencia de que todos los objetivos planteados en el trabajo se han cumplido correctamente.

Respecto a la evaluación de la asignatura, el diseño del autogiro necesita de la mayoría de los conocimientos estadísticos e informáticos que los alumnos debieran haber adquirido durante el curso, conectando y estructurando mentalmente dichos conocimientos hasta llegar al diseño final. La escritura del informe final necesita de un proceso de racionalización, y el dominio de un lenguaje específico de la materia, y la presentación pública del trabajo necesita de síntesis y adaptación a las necesidades de la audiencia. En nuestra opinión, este tipo de actividades se configura como una opción muy completa en la evaluación de los conocimientos de la materia. Sin embargo, siempre es necesaria una calificación numérica para incluirla en un acta de la asignatura, por lo que el éxito del cambio podremos medirlo al comparar los resultados que han obtenido nuestros alumnos en las seis ediciones en que la materia completa de DOE ha sido ofertada en el máster.

En la Tabla 2 presentamos el número de alumnos totales que han obtenido cada una de las cuatro calificaciones en la materia (Excelente, Notable, Aprobado, No Presentado) en cada uno de los seis años en los que se ha impartido la materia de DOE en el máster, y en la Figura 3 presentamos gráficamente estos mismos resultados. Resaltar que en la tabla aparecen unos pocos alumnos que figuran como no presentados. La causa es la obtención de un contrato laboral y la incompatibilidad de los horarios entre profesión y máster, o definitivamente trabajar en el extranjero. 
Tabla 2. Resultados de la evaluación en los seis años de impartición

\begin{tabular}{|ccccccc|}
\hline AÑO & Exc & Not & Apr & NP & Tot & Evaluación \\
\hline $\mathbf{2 0 0 9 - 2 0 1 0}$ & 0 & 2 & 5 & 1 & 8 & Trabajo \\
$\mathbf{2 0 1 1 - 2 0 1 2}$ & 0 & 5 & 17 & 1 & 23 & Trabajo \\
$\mathbf{2 0 1 3 - 2 0 1 4}$ & 0 & 4 & 0 & 0 & 4 & Trabajo \\
$\mathbf{2 0 1 5 - 2 0 1 6}$ & 8 & 4 & 0 & 0 & 12 & Experimento \\
$\mathbf{2 0 1 6 - 2 0 1 7}$ & 4 & 10 & 0 & 0 & 14 & Experimento \\
$\mathbf{2 0 1 7 - 2 0 1 8}$ & 5 & 2 & 0 & 1 & 8 & Experimento \\
\hline
\end{tabular}

Desde el inicio del máster, y hasta el curso 2013-2014, la evaluación de la materia se realizaba mediante un trabajo en el que los alumnos analizaban dos problemas reales, que obteníamos a través de artículos de investigación que eran publicados en revistas de ingeniería (referenciado como Trabajo en la Tabla 2). Los alumnos analizaban los artículos, en cuanto al diseño de experimentos utilizado y al análisis de los resultados del trabajo realizado por otros, repitiendo los análisis, y rechazando, corroborando o ampliando las conclusiones que en ellos se encontraban. Las notas obtenidas eran bastante discretas, con $67 \%$ de aprobados y un 33\% de notables. En el curso 2015-2016 se introdujo por fin el cambio en la metodología docente, con la inclusión de la experimentación real en el proceso de aprendizaje, cubriendo así el vacío existente entre el propio diseño del experimento y el análisis de los datos resultantes (referenciado como Experimento en la Tabla 2). Los resultados son muy satisfactorios, dado que el rango de las calificaciones obtenidas ha pasado del aprobado-notable al notable-excelente, con un 53\% de excelentes y un $47 \%$ de notables. Si consideramos que los alumnos son evaluados de un número considerablemente mayor de competencias, la mejora es todavía mucho mayor.

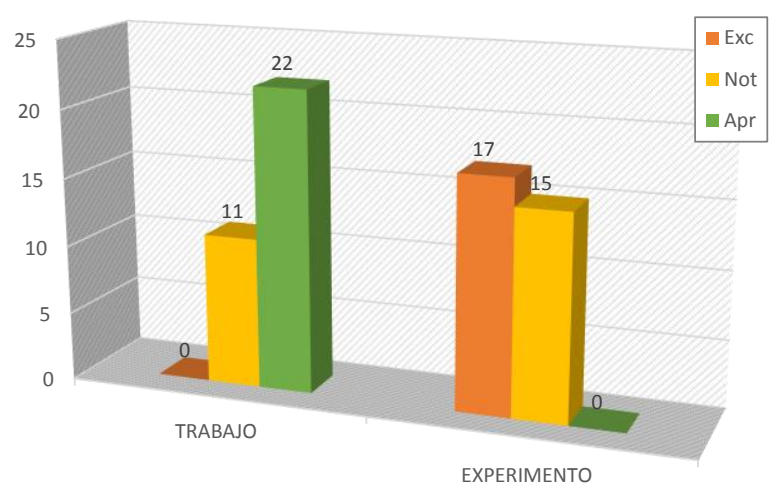

Fig. 3 Rendimiento de los alumnos en la materia de DOE en los seis años analizados, antes y después de la introducción de la innovación escogida 


\section{Conclusiones}

Cuando en el año 2009 comenzó el máster de Ingeniería de Análisis de Datos, Mejora de Procesos y Toma de Decisiones, el concepto de aprendizaje basado en competencias no existía como tal para la UPV. Sin embargo, la materia de DOE se trató de estructurar en torno a actividades que reflejaran su aplicación en la industria, y que involucraran la participación activa de los alumnos en el aprendizaje.

La aparición explícita en las guías de las asignaturas de las competencias que deben adquirir los alumnos, y especialmente las competencias transversales, ha permitido introducir cambios en la realización de las sesiones prácticas y en la evaluación de ambas asignaturas. La dirección del cambio resulta obvia, si se atiende al título de la materia que impartimos, pues nada mejor que los alumnos realicen experimentos reales en el laboratorio para desarrollar y evaluar las competencias que la ERT nos solicita, además de resaltar la importancia que tiene el diseño de experimentos en la industria.

Esta metodología activa refuerza los objetivos propuestos en ambas asignaturas: la selección del diseño de experimentos y su análisis mediante la herramienta estadística más adecuada, la interpretación de los resultados obtenidos y la determinación de las condiciones operativas óptimas del proceso o servicio en estudio. La actividad escogida permite estimular y motivar el trabajo y la participación activa del alumno en aula, permite desarrollar las competencias de trabajo en equipo, planificación, análisis y resolución de problemas, así como la redacción y presentación pública de informes de carácter técnicocientífico. Consideramos que el experimento propuesto ayuda a obtener una visión global de los contenidos de la materia, además de hacer explícitas y evaluables aquellas competencias que se han comprometido con la ERT, mejorando y potenciando el aprendizaje. Con la introducción de esta actividad pensamos que los alumnos formados serán mejores profesionales en su sector, contribuyendo a cumplir con las expectativas de formación que la sociedad actual demanda a la universidad. 


\section{Referencias}

Chirivella, V., Alcover, R. y Richart, B. (2018). Metodologías activas: simulación de un proceso de centrifugado en Diseño de Experimentos en ingeniería. En Actas del IV Congreso Nacional de Innovación Educativa y Docencia en Red (IN-RED 2018), pág. 1225-1237, Julio 2018, Valencia.

Engineering.com (2013). Disposable UAVs Inspired by Paper Planes. En https:/www.engineering.com/DesignerEdge/DesignerEdgeArticles/ArticleID/6016/DisposableUAVs-Inspired-by-Paper-Planes.aspx.

Fernández March, A. (2006). Metodologías activas para la formación de competencias. Educatio S.XXI núm. 24, pág. 35-56. En http://revistas.um.es/educatio/article/view/152/135.

Gima (2008). Metodologías activas. Valencia : Universitat Politècnica de València, Editorial UPV.

González, N. (2013). Metodologías activas en la docencia universitaria: importancia, conocimiento y uso en opinión del profesorado. REVALUE Revista de evaluación educativa, (vol. 2, núm. 2, pág. 88-107). En http://revalue.mx/revista/index.php/revalue/issue/current [Consulta: 18 de marzo de 2018].

Montgomery, D. C. (2012). Design and analysis of experiments. Ed. John Wiley \& Sons, New York.

Peiro-Signes, A. y Segarra-Oña, M. (2015). Experiential learning as a dynamizer of class activity. En Proceedings of International Conference of Education, Research and Innovation, pág. 1010-1013. Noviembre 2015, Sevilla.

Romero, R. y Zúnica, L.R. (2004). Métodos estadísticos en Ingeniería, Editorial Universitat Politècnica de València.

UPV. Proyecto institucional de las competencias transversales. En http://www.upv.es/contenidos/COMPTRAN/ [Consulta: 14 de marzo de 2018].

Villa, A. y Poblete, M. (2007). Aprendizaje basado en competencias. Una propuesta para la evaluación de las competencias genéricas. Ediciones Mensajero. Bilbao. 